\title{
CHARACTERISTICS OF HYBRID BROADCAST BROADBAND TELEVISION (HBBTV)
}

\author{
BRANIMIR JAKŠIĆ ${ }^{*}$, IVANA MILOŠEVIĆ ${ }^{2}$, MILE PETROVIĆ ${ }^{1}$, SINIŠA ILIĆ ${ }^{1}$, \\ SLOBODAN BOJANIĆ ${ }^{3}$, SELENA VASIĆ ${ }^{4}$
}

${ }^{1}$ Faculty of Technical Sciences, University of Priština, Kosovska Mitrovica, Serbia

${ }^{2}$ High School of Electrical Engineering and Computers, Belg rade, Serbia

${ }^{3}$ ETSI Telecomunicacion, Universidad Politecnica de Madrid, Madrid, Spain

${ }^{4}$ Faculty of Information Technology, University of Metropolitan, Belgrade, Serbia

\begin{abstract}
This paper describes the working principle of hybrid broadcast-broadband TV (Hybrid Broadcast Broadband TV - HbbTV). The architecture of HbbTV system is given, the principle of its operation, as well as an overview of HbbTV specification standards that are in use, with their basic char acteristics. Here are described the services provided by Hybrid TV. It is also provided an over view of the distribution of HbbTV services in Europe in terms of the number of TV channels that HbbTV services offer, the number of active hybrid TV devices, Hbb TV standards which are in use and models of broadcast networks used to distribute HbbTV service.
\end{abstract}

Keywords: Hybrid TV, HbbTV, broadcasting networks, broadband networks, digital terrestrial TV, cable, satellite, IPTV.

\section{INTRODUCTION}

The Internet has grown into a massive medium for delivering large amounts of data on various topics. Digitization of television, as second massive media, and the rapid development of the Internet lead to need to connect these two media. Hybrid broadcasting wideband television (Hybrid Broadcast Broadband TV - HbbTV) is a global initiative aimed at harmonizing standards for content delivery to the end user, while using, at the same time, the broadcasting (broadcast) and wideband (broadband) network via the linked TV devices (connected TV, Smart TV) and Set -Top-Boxes (HbbTV website, 2016), (Illinger, 2012). Actually, HbbTV is a technology that combines broadcasting services with services offered over the internet - all at one screen.

Unlike the traditional television, where the user is a passive observer, hybrid TV users can use interactive applications for entertainment or to obtain information.

A wide variety of additional services which Allows HbbTV can be divided into the following categories (Merkel, 2010):

- Voice subtitles, audio of other languages.

- E-Administration, "a digital counter" for the services of the national government or local authorities.

- "Super text" - an advanced teletext with a more attractive user interface with informations (news, weather, traffic, sports scores, stock market, etc.). Thanks to HTML (Hyper Text Markup Language) and connecting to the Internet, images, graphs, maps, and similarly can be displayed within the Super-text.
- Advanced Electronic Program Guide - EPG (Electronic Program Guides).

- Enhanced TV - Additional information on TV programs such as statistics in sports programs, adds with biographies, background events, etc.

- The vote, participation in TV shows, vote for candidates in shows and other.

- Additional services such as "catch up TV" - delayed viewing of the TV programs, restoration of the TV program, video on demand (VOD - Video on Demand), picture in picture and other.

- Direct access to additional TV channels which are not represented in cable systems, digital terrestrial or satellite television via live streaming (over 15,000 channels worldwide).

- Simple games on television.

- Home Shopping, courses, tele-education.

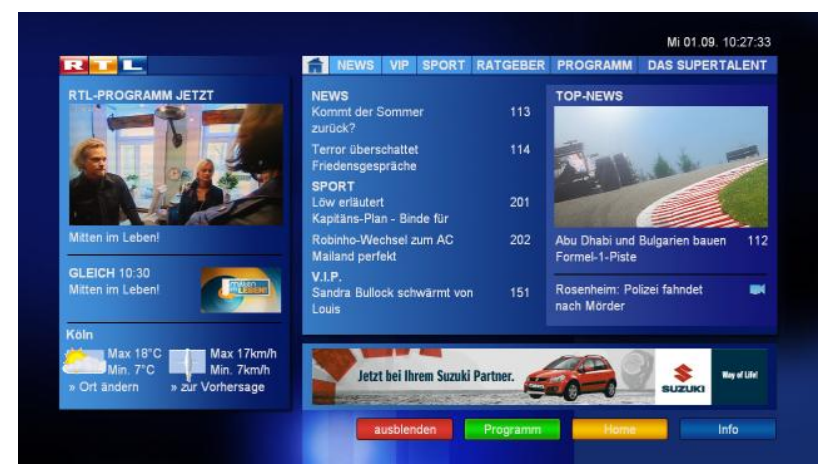

Fig. 1. HbbTV applications of RTL Germany. 


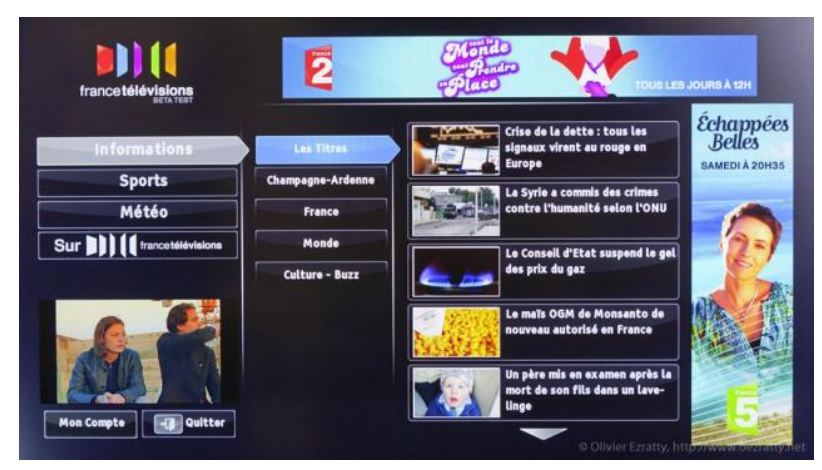

Fig. 2. HbbTV applications of France 2 TV.

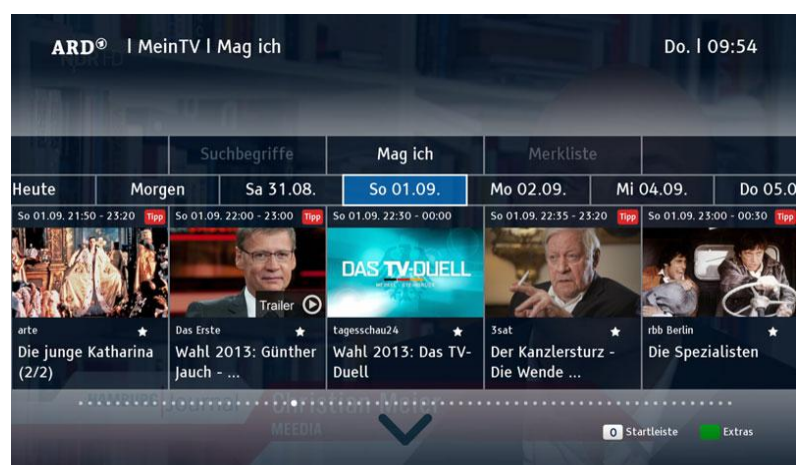

Fig. 3. Advanced EPG with in the HbbTV applications of German ARD.
In Fig. 1 (He ise website, 2016), Fig. 2 (Forumdesforums website, 2016) and Fig. 3 (Parabola website, 2016), is given appereance of applications HbbTV RTL, France 2 and ARD TV.

\section{ARCHITECTURE OF HBB TV S YS TEM}

HbbTV uses two networks - broadcasting and broadband to deliver data and applications on hybrid devices (terminals). Typical hybrid devices are Internet-connected (Internetconnected) TV sets and Set-Top Boxes, computers with tuners and mobile phones with broadcast receivers. Today, many manufacturers of consumer electronics offer Internet-connected TVs and Set-Top-Boxes. There are different names for these solutions, such as Smart TV and NetTV (Clover, 2009).

Hybrid terminal has the ability to be connected to the two networks in parallel. On the one hand it can be one of broadcast DVB network (terrestrial DVB-T, DVB-S satellite or cable DVB-C). Through this broadcasting connection the hybrid terminal can receive the standard broadcasting A / V (ie. A linear A / V content), applications and signaling. Even if the terminal is not connected to a broadband network, its connection with the broadcasting network allows it to receive feasts applications. In addition, the signaling stream events in the application are possible via the broadcasting network.

\section{Broadcast}

\section{Broadband}

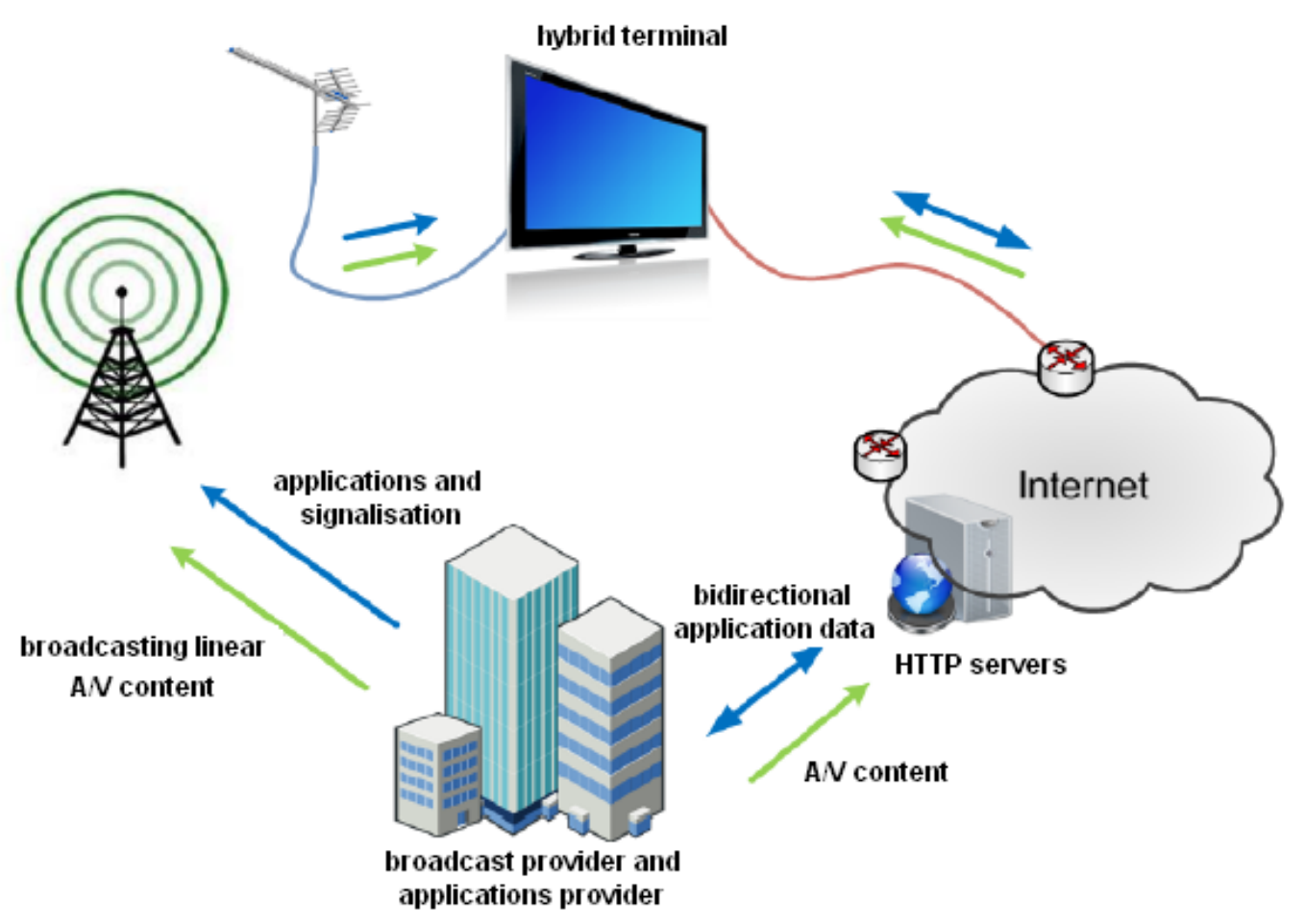

Fig. 4. Architecture of HbbTV system. 
The hybrid terminal can be connected to the Internet via a broadband interface. This connection allows two-way communication with the provider applications. Through this interface terminal can receive the applications and the non-linear A / V content (e.g., streaming A / V content on the request). The hybrid terminal can support download A / V content is independent of the weather. Through broadband interface, the terminal can also be connected to other HbbTV terminals in the same local area network (HbbTV website, 2015).

Fig. 4 shows the architecture of a system with a hybrid terminal connected with the DVB-T network as an example of the broadcasting connection.

\section{HBB TV SPECIFICATIONS}

HbbTV standards are developed by the HbbTV Association, and published by the ETSI (European Telecommunications Standardization Institute). HbbTV Association was established in February 2009.

First demonstration of HbbTV was in 2009 in France by France Télévisions and two makers of Set-Top-Box technology, Inverto Digital Labs, Luxembourg, PLEYO from France, during Roland Garros using DTT transmission and IP connections and in Germany using satellite Astra $19.2^{\circ}$ east, during IFA and the IBC exhibitions.

In June 2014, HbbTV Association is connected to the Open IPTV Forum (IPTV Forum website, 2016), a similar industrial organization for Internet Protocol television (IPTV) services established in 2007, which has worked closely with the HbbTV initiative on specification network connected TVs and Set-Top Boxes. Activities of Open IPTV Forum are transferred to the jurisdiction of the HbbTV Association. This extended the jurisdiction of HbbTV Association includes defining specifications for providers services which make it easier to accelerate the imple mentation of IPTV services

HbbTV Specification Version 1.0 (HbbTV specifications, 2010), was approved by ETSI as ETS TS 796, in June, 2010. In Table 1, are given the lables of HbbTV specification standards and appropriate ETSI approvals (HbbTV website, 2016).

Table 1. HbbTV Specifications.

\begin{tabular}{|l|l|l|}
\hline $\begin{array}{l}\text { HbbTV } \\
\text { specificati ons }\end{array}$ & ETS I approval & Year \\
\hline HbbTV 1.0 & TS 102796 v1.1.1 & June, 2010. \\
\hline HbbTV 1.5 & TS 102796 v1.2.1 & November, 2012. \\
\hline HbbTV 2.0 & TS 102796 v1.3.1 & November, 2015. \\
\hline
\end{tabular}

HbbTV specification is built on existing standards and web technologies including OIPF (Open IPTV Forum), Consumer Technologies Association (CTA website, 2016), DVB-Digital Video Broadcasting) (DVB website, 2016) and the W3C (World Wide Web Consortium website, 2016). Standard provides the features and functionality which is required to meet the standards of good broadcasting and Internet services. By using the standards of Internet technologies, rapid development of applications is enabled.

HbbTV specification with label 1.5 provides the features and functionality needed to deliver a variety of services for broadcasting. HbbTV specification 1.5 introduces support for HTTP adaptive streaming based on MPEG-DASH specification, which improves video quality on too burdened or slow internet connections. Version 1.5 greatly improves access to informations which are related to the television program given by providers via EPG.

HbbTV Association has announced in 2015 a new HbbTV 2.0 specification. HbbTV Association expects that the manufacturers, broadcasters and operators will start to introduce a new generation of hybrid interactive TV service in 2016 (HbbTV website, 2016).

With HbbTV 2.0, consumers will be able to enjoy a wide range of new services, including (HbbTV website, 2016):

- Advanced user applications based on the HTML5.

- Watching video content via smartphone, PC and tablet devices.

- Advanced supporting applications about detailed review of the program, voting, games and more.

- Standardized delivery of Ultra HD content using HEVC compression standard.

- Improvement of services with better support for translation in multiple languages.

- Access to broadcasting content recorded on the hard disk of the receiver.

- Support for consumer privacy.

To achieve all these benefits, the new specification includes a series of new technologies, including support for HTML5, DVB CI Plus 1.4, HEVC video and TTML subtitles (Timed Text Markup Language). It also improves support for existing technologies, including MPEG DASH, DSM-CC (Digital Storage Media Command and Control) and synchronization applications on the TV. It is designed to ensure that HbbTV HbbTV 1.0 and 1.5 applications continue to work on the HbbTV 2.0 devices.

\section{HBB TV IN EUROPE}

At the moment HbbTV is the most developed in Germany, France and Spain. HbbTV is started in the German public service broadcasters - ARD and ZDF and France - France Télévision. Other countries where the HbbTV services are regularly provided are: United Kingdom, Belgium, Netherlands, Switzerland, Austria, Poland, Czech Republic, Denmark and Finland, and since 2016, and Norway and Sweden (Girons, 2015). Fig. 5 gives the situation in broadcasting HbbTV services in European countries on 1 January 2016. 


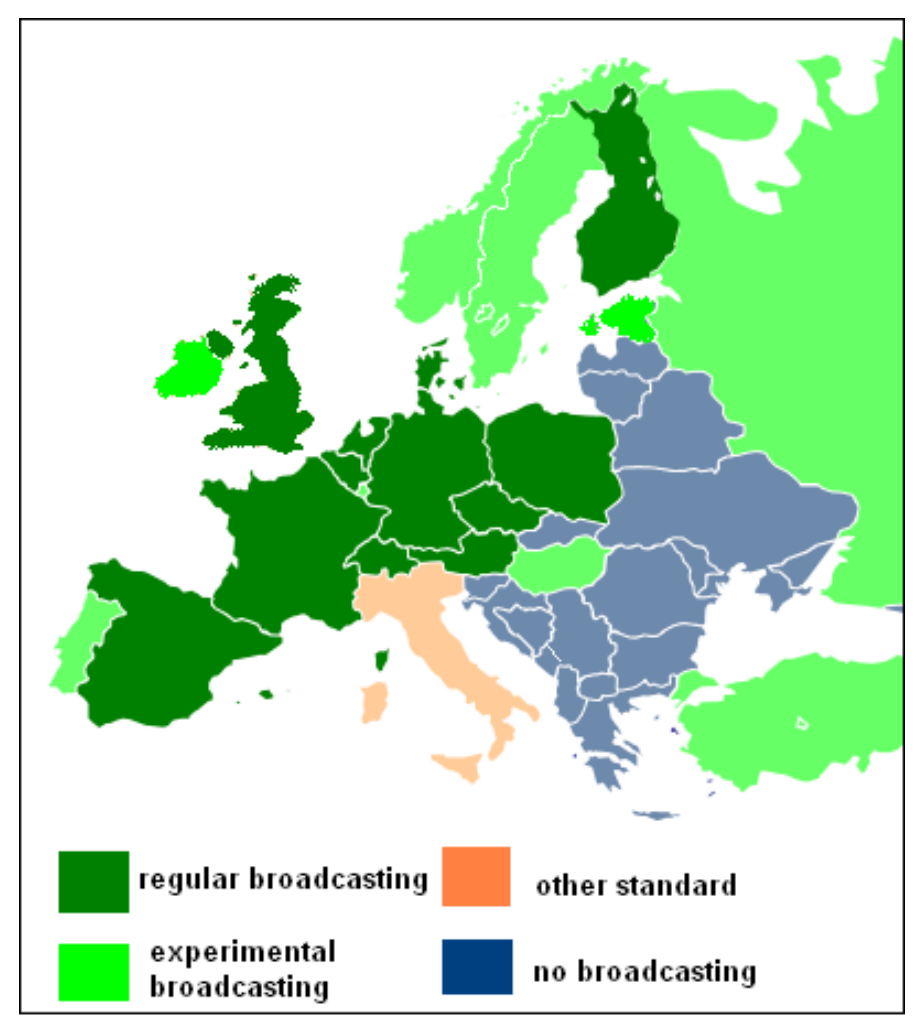

Fig. 5. Broadcast HbbTV services in Eu rope.

In European countries, forms of distribution of TV programs are variously represented. Table 2 contains the representation in\% of digital terrestrial TV (DTT - Digital terrestrial television), satellite, cable, and IPTV in the distribution of TV channels to end-users in the second half of 2015 (Girons, 2015). The most common form of distribution in one country entails that the HbbTV services are most used in that system (broadcasting network).

Table 2. Representation of Terrestrial, Satellite, Cable and IPTV Systems in Europe

\begin{tabular}{|c|c|c|c|c|c|}
\hline Country & $\begin{array}{c}\text { Househol ds } \\
{[\text { million] }}\end{array}$ & $\begin{array}{c}\text { DTT } \\
{[\%]}\end{array}$ & $\begin{array}{c}\text { Satellite } \\
{[\%]}\end{array}$ & $\begin{array}{c}\text { Cable } \\
{[\%]}\end{array}$ & $\begin{array}{c}\text { IPTV } \\
{[\%]}\end{array}$ \\
\hline Germany & 38.5 & 9 & 43 & 43 & 4 \\
\hline France & 27.0 & 42 & 18 & 6 & 34 \\
\hline Spain & 19.0 & 81 & 4 & \multicolumn{2}{|c|}{15} \\
\hline Austria & 3.6 & 6 & 53 & \multicolumn{2}{|c|}{41} \\
\hline Poland & 13.5 & 28 & 54 & 16 & 2 \\
\hline Netherlands & 17.0 & 6 & 6 & 62 & 26 \\
\hline Switzerland & 3.3 & 3 & 10 & 56 & 31 \\
\hline Czech Rep. & 4.5 & 44 & 38 & 13 & 5 \\
\hline Den mark & 5.6 & 14 & 9 & 74 & 3 \\
\hline Fin land & 2.4 & 46 & 3 & 43 & 8 \\
\hline Sweden & 4.5 & 25 & 15 & 50 & 10 \\
\hline Norway & 2.2 & 10 & 31 & \multicolumn{2}{|c|}{59} \\
\hline Hungary & 4.2 & 18 & 23 & 55 & 4 \\
\hline
\end{tabular}

The first HbbTV services in Germany started its work in 2010. Number of HbbTV devices in use in Germany in 2015 amounted to 12 million. HbbTV services are available in all broadcast networks (satellite, cable, terrestrial and IPTV) with 75 TV channels. Included standard is HbbTV 1.0 (Girons, 2015).

In France, the HbbTV services regularly begin to work in 2011. Number of active HbbTV devices in 2015 was about one million. HbbTV services are available in all broadcasting networks. In use are the standards HbbTV 1.0 and 1.5. Currently, the HbbTV service is available in about 15 TV channels. MyTF1 VOD is the first TNT2.0 service based on HbbTV 1.5 using MPEG-DASH adaptive streaming (Girons, 2015).

In Spain HbbTV services began to operate in 2013. Nearly 1.5 million HbbTV devices are in use in 2015. HbbTV services are the most represented in digital terrestrial TV and in $15 \mathrm{TV}$ channels. In use is HbbTV standard 1.5. TDT Hibrida is the Spanish HbbTV DTT 1.5 specification.

The UK has a hybrid services that are broadcast through the MHEG-5.United Kingdom launched interactive television with the included MHEG-5 in its digital terrestrial TV since 2012. Since October 2015, launched the services Freeview Play platform by using standard HbbTV 2.0. A mong the TV channels which provide these hybrid services are BBC, Channel 4, Channel 5, ITV and others. The service is available within the digital terrestrial and satellite TV.

In Poland HbbTV services are available in all broadcasting networks. In use is an upgraded version of the standard HbbTV 1.0 - 1.1 HbbTV. All public and a large number of TV channel offers HbbTV services (Dziadul, 2015).

In Austria, all HbbTV services are available for all types of broadcast networks. HbbTV services are available in $6 \mathrm{TV}$ channels, as well as in the German TV channels available via satellite. They use about one million HbbTV devices. Used HbbTV 1.0 standard (ORF website, 2015).

In Belgium, the HbbTV services are available through the Walloon RTBF in the digital terrestrial network (DTT).

Dutch broadcasters for distributing HbbTV services are using standard HbbTV 1.5. The most significant emitters are NSO and SPS.

In Switzerland in 2015. were already 2 million HbbTV devices. In use is the HbbTV standard 1.0, and 1.5 and 2.0 are tested. HbbTV services provide all public broadcasters (6 TV channels in French, Italian and German), as well as other German and French broadcasters. HbbTV services are distributed through satellite and cable systems (Girons, 2015).

Broadcasters in the Czech Republic for distribution HbbTV services using standard HbbTV 1.0 (RTS website, 2014). During 2015, there were 740,000 hybrid connected TV devices.

In Denmark, the distribution of HbbTV services uses standard HbbTV 1.1 and services are available in cable, terrestrial and IPTV systems. During 2015, there were 240,000 active hybrid TV devices. 
In Finland, the distribution of HbbTV services uses standard HbbTV 1.1 and HbbTV 1.5. HbbTV services are mostly represented in the digital terrestrial TV (DTT) (Sofiadigital website, 2015).

In Norway and Sweden HbbTV services will regularly begin with operations during 2016. Since 2014. They are in the test phase, and for distribution HbbTV services uses the standard HbbTV 1.5.

In Hungary HbbTV services are provided by Antenna Hungaria (Antenna Hungária website, 2016) in the digital terrestrial network, using standard HbbTV 1.0. In 2015, there were 200,000 active HbbTV devices.

Russia has been got in 2013., within the DVB-T2 network, the first HbbTV services. HbbTV services are available in some pay TV operators (RTS website, 2013).

In Estonia, the HbbTV services were launched in the beginning of 2015. in the context of digital terrestrial TV (RTS website, 2015).

In Italy is not in use HbbTV standard, for Interactive TV is used MHP (Multimedia Home Platform) Hybrid TV. It was developed by the DVB Project in 2000. MHP is a Java-based middleware system orig inally designed for telephone line with interactive channels. Fast internet now allows that potential of MHP can be fully realized. Interactive $\mathrm{TV}$ has been a fundamental element for the launch of digital terrestrial television in Italy and MHP was at the center of this offer.

\section{CONCLUSION}

The introduction of the Hybrid Broadcast Broadband TV (HbbTV) enabled offering a wide range of interactive services to the end users. HbbTV provides a direct connection between the linear programs and online content. HbbTV is directly implemented at the so-called. "Connected TV" or appropriate Set-Top-Boxes, without purchasing additional equipment. Providers only need to develop applications in accordance with one of the HbbTV standard, instead of creating different versions of applications for each model of TV or for each model of SetTop-Box. A large number of European countries included HbbTV services and the most common are in Germany, France, Spain, Austria and Poland. During 2015, more than 90\% of all manufacturers of TV equipment have adopted the HbbTV standard (Gesellschaft für Konsumforschung website, 2016). It is estimated that in 2016 more than $50 \%$ of all TVs available in homes in Europe will be compatible for HbbTV.

\section{ACKNOWLEGMENTS}

This work was done within the Erasmus Plus CapacityBuilding projects in the field of Higher Education: "Implementation of the Study Program - Digital Broadcasting and Broadband Technologies (Master Studies)", Project No. 561688-EPP-1-2015-1-XK-EPPKA2-CBHE-JP.

\section{REFERENCES}

-Antenna Hungária. (2016). . website. Retrieved from www.ahrt.hu

Clover, J. 2009. EBU General Assembly backs HBB.Broadband TV News.

-Consumer Technology Association (CTA). (2016). . website. Retrieved fro mhttps://www.cta.tech/

-Digital Video Broadcasting (DVB). (2016). . website. Retrieved from https://www.dvb.org/

Dziadul, C. 2012. HbbTV starts in Poland.Broadband TV News.

-Forumdesforums. 2016. Forumdesforums website. Retrieved from

http://www.foru mdesforums.co m/modules/news/article.php?s toryid $=62067$

-Gesellschaft für Konsumforschung (Gfk SE). (2016). . website. Retrieved from www.gfk.com

Girons, R.S. 2015. Country Review Europe. . In: HbbTV Symposiu m, 2015-12-08, London.

-European Telecommunications Standards Institute (ETSI). 2010. HbbTV specifications. TS 102796 V1.1.1..

-HbbTV Association. 2015. HbbTV 2.0 Specification. Retrieved from https://www.hbbtv.org/wpcontent/uploads/2015/07/HbbTV_specification_2_0.pdf

-HbbTV. (2016). . website. Retrieved from http://www.hbbtv.org/

-He ise. 2016. RTL startet HbbTV Regelbetrieb mit HD. website. Retrieved from

http://www.heise.de/newsticker/meldung/RTL-startet

HbbTV-Regelbetrieb-mit-HD-Videotext 1070641 .ht ml? view=zoom; zoo $\mathrm{m}=1$

Illgner, K. 2012. HbbTV Road map.HbbTV Consortiu m.

Merkel, K. 2010. HbbTV: A hybrid broadcast-broadband system for the living room.Munich: IRT.

-Open IPTV Forum. (2015). . website. Retrieved from www.oipf.tv

-Österreich ischer Rundfunk (ORF). (2015). . website. Retrieved from www.orf.at

-Parabola. (2016). . website. Retrieved from http://www.parabola.c z/clanky/4738/hbbtv-jiz-take-napristrojich-vu-plus/

-RTS. 2013. HbbTV platforma u Rusiji. website. Retrieved from www.rts.rs/page/rts/sr/Digitalizacija/story/1578/Svet/145305 3/HbbTV+platforma+u+Rusiji.html

-RTS. 2014. HbbTV napreduje u Češkoj. website. Retrieved from http://www.rts.rs/page/rts/sr/Digitalizacija/story/1578/Svet/17 70379/HbbTV+napreduje+u +\% C4\% 8Ce\% C5\% A 1 koj.html

-RTS. 2015. HbbTV stiže u Estoniju. website. Retrieved from http://www.rts.rs/page/rts/sr/Digitalizacija/story/1578/Svet/18 57203/HbbTV+sti\% C5\% BEe+u+Estoniju.html

-Sofiadigital. 2015. Finland is setting the standard for connected TV services. website. Retrieved from http://s ofiadigital.co $\mathrm{m} /$ finland-is-s etting-the-standard-forconnected-tv-services/

-World Wide Web Consortium. (2016). . website. Retrieved from https://www.w3.org/ 